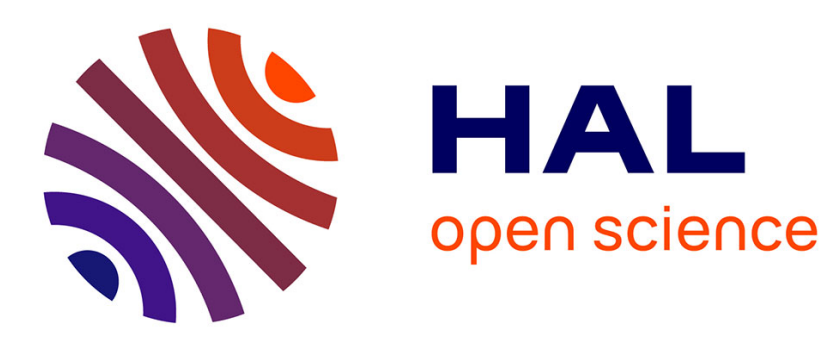

\title{
Extended output depending normal form
}

\author{
Ramdane Tami, Driss Boutat, Gang Zheng
}

\section{To cite this version:}

Ramdane Tami, Driss Boutat, Gang Zheng. Extended output depending normal form. Automatica, 2013, 49, 7, pp.2192-2198. 10.1016/j.automatica.2013.03.025 . hal-00817332

\section{HAL Id: hal-00817332 \\ https://inria.hal.science/hal-00817332}

Submitted on 8 Mar 2017

HAL is a multi-disciplinary open access archive for the deposit and dissemination of scientific research documents, whether they are published or not. The documents may come from teaching and research institutions in France or abroad, or from public or private research centers.
L'archive ouverte pluridisciplinaire HAL, est destinée au dépôt et à la diffusion de documents scientifiques de niveau recherche, publiés ou non, émanant des établissements d'enseignement et de recherche français ou étrangers, des laboratoires publics ou privés. 


\title{
Extended output depending normal form
}

\author{
Ramdane Tami ${ }^{\mathrm{a}, 1}$, Driss Boutat ${ }^{\mathrm{a}}$, Gang Zheng ${ }^{\mathrm{b}}$ \\ ${ }^{a}$ Loire Valley University, ENSI-Bourges, Laboratoire PRISME, 88 Bd. Lahitolle, 18020 Bourges Cedex, France. \\ ${ }^{b}$ INRIA Lille-Nord Europe, 40 Avenue Halley 59650.
}

\begin{abstract}
This paper concerns the design of a nonlinear observer through a transformation of a nonlinear system into an observer form that supports a high gain observer. Sufficient geometrical condition is deduced to guarantee the existence of change of coordinates allowing the transformation of a nonlinear system into the proposed normal form. The result is highlighted by studying the Susceptible Exposed Infected and Recovered (SEIR) model.
\end{abstract}

Keywords: Extended Normal Form, Nonlinear system, Observer, Epidemic model.

\section{Introduction}

The observer design for nonlinear dynamical systems is an important issue in the control theory. Several approaches are proposed to design nonlinear observers for many cases, while one of the methods is to transform the nonlinear system into a more simple form which enables to apply existing observers.

The first idea of normal form is due to Bestle and Zeitz (1983) for time variant dynamical systems, and to Krener and Isidori (1983) for time invariant dynamical systems, where the author introduced the so-called observer canonical form with output injection with all nonlinear terms being only function of the output. Then Krener and Respondek (1985) gave the associated canonical form with output injection for multi-outputs nonlinear systems without inputs, and the result for multi-outputs systems with inputs was studied in Xia and Gao (1989). Based on the above works many algorithms are developed to generalize the existing results, including algebraic approaches (Keller (1987); Phelps (1991); Rudolph and Zeitz (1994)), geometrical approaches (Hou and Pugh (1999); Marino and Tomei (1996); Boutat et al. (2009); Lynch and Bortoff (2001)) and the so called direct transformations stated in Lopez et al. (1999). To enlarge the class of observer forms, the concept of output depending normal form was firstly addressed in Guay (2002); Respondek et al. (2004), then was developed in Zheng et al. (2007) and Wang and Lynch $(2009,2010)$. The other approaches to enlarge the class of normal forms are the extended normal form introduced in (Jouan (2003); Noh et al. (2004); Back et al. (2006); Yang et al. $(2010,2011)$ ), where the main idea is to add an auxiliary dynamics to the dynamical system in such a way that the extended system can satisfy the conditions proposed in the literature. The geometrical characterization of the

Email addresses: ramdane.tami@ensi-bourges.fr (Ramdane Tami), driss.boutat@ensi-bourges.fr (Driss Boutat), gang.zheng@inria.fr (Gang Zheng)

${ }^{1}$ This work was supported by Région Centre France second method was addressed in Boutat (2007) and Boutat and Busawon (2011).

In this paper, we propose a new observer normal form by mixing the output depending normal form and the extended normal form. Precisely, we give geometrical sufficient condition that guarantees the transformation of a dynamical system via a change of coordinates to a new extended observer normal form. Then, we apply this method to the Susceptible Exposed Infected and Recovered (SEIR) model.

This paper is organized as follows. Section 2 presents motivation example and the problem statement. In section 3 , a new extended output depending normal form is proposed, and sufficient condition is established allowing the transformation of nonlinear dynamical systems into the proposed normal form. Section 4 applies the proposed result to study SEIR model.

\section{Preliminaries}

\subsection{Geometrical background}

Herein, we introduce a background on some geometrical tools that will be needed afterward. Consider a single output nonlinear dynamical system in the following form:

$$
\begin{aligned}
& \dot{x}=f(x) \\
& y=h(x)
\end{aligned}
$$

where $x \in U \subseteq \mathbb{R}^{n}$ is the state and $y \in \mathbb{R}$ is the output. We assume that the vector field $f$ and the output function $h$ are smooth. In the following, we also assume that the pair $(h, f)$ satisfies the observability rank condition. Thus, the so-called observability differential 1-forms are independent and are given by:

$$
\begin{aligned}
& \theta_{1}=d h \\
& \theta_{i}=d L_{f}^{i-1} h \quad \text { for } 2 \leq i \leq n
\end{aligned}
$$

where $L_{f}^{k} h$ is the $k^{t h}$ Lie derivative of $h$ along $f$ and $d$ is the differential operator. 
Thus, one can construct the Krener and Isidori (1983) frame $\tau=\left[\tau_{1}, \cdots, \tau_{n}\right]$ where the first vector field $\tau_{1}$ is a solution for the following algebraic equations:

$$
\begin{aligned}
\theta_{i}\left(\tau_{1}\right) & =0 \quad \text { for } 1 \leq i \leq n-1 \\
\theta_{n}\left(\tau_{1}\right) & =1
\end{aligned}
$$

and the other vector fields are given by induction as follows:

$$
\tau_{i}=\left[\tau_{i-1}, f\right]
$$

for $2 \leq i \leq n$, where [, ] denotes the Lie bracket.

In Krener and Isidori (1983), the commutativity of Lie bracket, i.e. $\left[\tau_{i}, \tau_{j}\right]=0$ for $1 \leq i \leq n, 1 \leq j \leq n$ is the necessary and sufficient condition to transform the system (1) into a nonlinear observer form with output injection. However, if this condition is not fulfilled, then another frame $\bar{\tau}=\left[\bar{\tau}_{1}, \bar{\tau}_{2} \ldots \bar{\tau}_{n}\right]$ can be built from $\tau$ according to Respondek et al. (2004); Guay (2002), Zheng et al. (2007), and Wang and Lynch (2009, 2010) as follows:

$$
\left\{\begin{array}{l}
\bar{\tau}_{1}=\pi \tau_{1} \\
\bar{\tau}_{i}=\frac{1}{\alpha_{i}}\left[\bar{\tau}_{i-1}, f\right]
\end{array}\right.
$$

where $\pi=\prod_{i=2}^{n} \alpha_{i}$, and $\alpha_{i}(y)$ for $2 \leq i \leq n$ being non vanishing functions of the output to be determined. If the commutativity of Lie bracket condition is fulfilled for the new frame $\bar{\tau}$, then the system (1) can be transformed into the output depending nonlinear observer form with output injection. However, there exist as well some dynamical systems which do not fulfill the above conditions, and this will be highlighted in the following section.

\subsection{Motivating example: SEIR model}

The following example motivates the research of this work. Let us consider the Susceptible Exposed Infected and Recovered (SEIR) model Iggidr (2006):

$$
\begin{aligned}
& \frac{d S}{d t}=b N-\mu S-\beta \frac{S I}{N}-p b E-q b I \\
& \frac{d E}{d t}=\beta \frac{S I}{N}+p b E+q b I-(\mu+\varepsilon) E \\
& \frac{d I}{d t}=\varepsilon E-(r+\delta+\mu) I \\
& \frac{d R}{d t}=r I-\mu R \\
& \frac{d N}{d t}=(b-\mu) N-\delta I
\end{aligned}
$$

where $S(t)$ is the susceptibility of the host population to the contagious disease, $E(t)$ is the exposed population but not yet expressing symptoms, $I(t)$ is the infected population, $R(t)$ is the recovered population, $b$ is the rate of the natural birth, $\mu$ is the rate of fecundity, $\beta$ is the transmission rate, $\delta$ is the death rate related to diseases, $\varepsilon$ is the rate at which the exposed population becomes infective, $p$ is the rate of the offspring from an exposed population, $q$ is the rate of the offspring from an infected population and $r$ is the rate at which the infected individuals are recovered.
It is supposed that one can measure the infected population $I(t)$ and the total population $N$ which is given as follows:

$$
N=S+E+I+R
$$

One wants to estimate the susceptibility of the host population $S(t)$ and the exposed population $E(t)$ from the infectious population. Then $R(t)$ can be deduced form the algebraical equation (8).

For the sake of simplicities, let us consider the normalized model of (7), by setting $x_{1}=\frac{S}{N}, x_{2}=\frac{E}{N}, x_{3}=\frac{I}{N}, x_{4}=\frac{R}{N}$ and $y=\frac{I}{N}$. Consequently the SEIR dynamics can be rewritten as follows:

$$
\begin{aligned}
\dot{x}_{1} & =b-b x_{1}+\gamma_{1} x_{1} x_{3}-p b x_{2}-q b x_{3} \\
\dot{x}_{2} & =\beta x_{1} x_{3}+\gamma_{2} x_{2}+\delta x_{2} x_{3}+q b x_{3} \\
\dot{x}_{3} & =\varepsilon x_{2}+\gamma_{3} x_{3}+\delta x_{3}^{2} \\
\dot{x}_{4} & =r x_{3}-b x_{4}+\delta x_{3} x_{4} \\
y & =x_{3}
\end{aligned}
$$

with $\gamma_{1}=-(\beta-\delta), \gamma_{2}=-(b+\varepsilon-p b), \gamma_{3}=-(r+\delta+b)$ and

$$
x_{1}+x_{2}+x_{3}+x_{4}=1
$$

We will show later in this paper that the system (9-11) with the output $y=h(x)=x_{3}$ does not fulfill any geometrical condition existing in the literature which allow to transform it into some existing observer forms. Therefore, this paper proposes a more general observer form that covers the existing forms proposed in Krener and Isidori (1983), Respondek et al. (2004); Guay (2002), Zheng et al. (2007)and Wang and Lynch (2009, 2010).

Inspired by the techniques of immersion and output injection, one can add the following auxiliary dynamics:

$$
\dot{w}=-\kappa(w)\left(\gamma_{1} y-b\right)
$$

where $w \in \mathbb{R}$ is an auxiliary variable, considered as an extra output and $\kappa(w)$ can be freely chosen in order to ensure the boundedness of $w$. Then the following diffeomorphism:

$$
\begin{aligned}
z_{1} & =\frac{\beta \varepsilon}{l(w)} x_{1}+\frac{b p \beta}{l(w)} x_{3} \\
z_{2} & =\frac{\varepsilon}{l(w)} x_{2}-\frac{\left(b+\gamma_{2}\right)}{l(w)} x_{3}-\frac{1}{2} \frac{\left(\delta-\gamma_{1}\right)}{l(w)} x_{3}^{2} \\
z_{3} & =\frac{1}{l(w)} x \\
\xi & =w
\end{aligned}
$$

where $l(w)=e^{\int_{0}^{w} \frac{b}{\kappa_{2}(s)} d s}$ determined in Section 4 and $\kappa_{2}(w)=$ $-\kappa(w) b$, allows the transformation of the system (9-11) and the auxiliary dynamics (15) into the following extended output depending form:

$$
\begin{aligned}
\dot{z}_{1} & =B_{1}(w, y) \\
\dot{z}_{2} & =y z_{1}+B_{2}(w, y) \\
\dot{z}_{3} & =z_{2}+B_{3}(w, y) \\
\dot{\xi} & =B_{4}(w, y) \\
\bar{y} & =z_{3}
\end{aligned}
$$


where $B_{i}$ for $1 \leq i \leq 3$ are given in Section 4 . For this transformed form, it can be seen that one can design a high-gain observer to estimate $z_{1}$ and $z_{2}$ with the known variables $z_{3}$ and $w$.

Consequently, in order to implement this new method, one should firstly propose a new normal form which mixes the immersion and output depending observer form, and then determine the auxiliary dynamics and the condition guaranteeing the existence of a diffeomorphism such that the system with immersion can be transformed into the proposed observer form.

\section{Main results}

\subsection{Extended output depending normal form and observer de- sign}

Consider the nonlinear system (1), one seeks an auxiliary dynamic $\dot{w}=\eta(y, w)$ so that the following extended dynamical system:

$$
\begin{aligned}
\dot{x} & =f(x) \\
\dot{w} & =\eta(y, w) \\
y & =h(x)
\end{aligned}
$$

could be transformed via a diffeomorphism $\left(z^{T}, \xi\right)^{T}=\phi(x, w)$ into the following more general extended output depending observer form:

$$
\begin{aligned}
\dot{z} & =A(y) z+B(y, w) \\
\dot{\xi} & =B_{n+1}(y, w) \\
y & =C z
\end{aligned}
$$

where $\xi \in \mathbb{R}, w \in \mathbb{R}, C=[0, \ldots, 0,1]$,

$$
A(y)=\left(\begin{array}{ccccc}
0 & \ldots & \ldots & \ldots & 0 \\
\alpha_{2}(y) & 0 & \ldots & \ldots & 0 \\
0 & \alpha_{3}(y) & \ldots & \ldots & \ldots \\
0 & \ldots & \ldots & \ldots & \ldots \\
0 & \ldots & \ldots & \alpha_{n}(y) & 0
\end{array}\right)
$$

The proposed form (21-23) supports the high-gain observer studied in Busawon et al. (1998) as follows:

$$
\begin{aligned}
\dot{\hat{z}} & =A(y) \hat{z}+B(w, y)-\Gamma^{-1}(y) R_{\rho}^{-1} C^{T}(C \hat{z}-\bar{y}) \\
0 & =\rho R_{\rho}+G^{T} R_{\rho}+R_{\rho} G-C^{T} C
\end{aligned}
$$

where

$$
\begin{aligned}
& G=\left(\begin{array}{cccc}
0 & \cdots & 0 & 0 \\
1 & \cdots & 0 & 0 \\
\vdots & \ddots & \vdots & \vdots \\
0 & \cdots & 1 & 0
\end{array}\right) \\
& \Gamma(y)=\operatorname{diag}\left[\prod_{i=2}^{n} \alpha_{i}(y), \prod_{i=3}^{n} \alpha_{i}(y), \cdots, \alpha_{n}(y), 1\right] \\
& R_{\rho}(n+1-i, n+1-j)=\frac{(-1)^{i+j} C_{i+j-2}^{j-1}}{\rho^{i+j-1}}
\end{aligned}
$$

for $1 \leq i \leq n$ and $1 \leq j \leq n$, where $C_{n}^{p}=\frac{n !}{(n-p) ! p !}$ is a binomial coefficient. The observation error dynamics will be governed by the following dynamics:

$$
\dot{e}=\dot{\hat{z}}-\dot{z}=\left(A(y)-\Gamma^{-1}(y) R_{\rho}^{-1} C^{T} C\right) e
$$

If $y$ and $w$ are bounded, then the observation error dynamics is exponentially stable by well choosing $\rho$.

\subsection{Determination of auxiliary dynamics and diffeomorphism}

In this section, we discuss the sufficient geometrical condition which guarantees the existence of an auxiliary dynamics $\dot{w}=\eta(y, w)$ and a diffeomorphism $\left(z^{T}, \xi\right)^{T}=\phi(x, w)$ for the purpose of transforming the extended system (18-20) into the proposed extended output depending observer normal form (2123) where $\xi \in \mathbb{R}$ and $w \in \mathbb{R}$.

For this, let us consider a function $l(w) \neq 0$ to be determined later and build the following new frame $\sigma$ from $\bar{\tau}$ defined in (6):

$$
\left\{\begin{array}{l}
\sigma_{1}=l(w) \bar{\tau}_{1} \\
\sigma_{k}=\frac{1}{\alpha_{k}}\left[\sigma_{k-1}, F\right]
\end{array}\right.
$$

where $\alpha_{k}$ for $2 \leq k \leq n$ is uniquely determined when constructing $\bar{\tau}$, and $F$ is the vector field for the extended system (18-20), noted as $F=f+\eta(y, w) \frac{\partial}{\partial w}$. Then we can state the following theorem.

Theorem 1. If there exists a function $l(w) \neq 0$ such that

$$
\left[\sigma_{i}, \sigma_{j}\right]=0
$$

for $1 \leq i \leq n$ and $1 \leq j \leq n$ where $\sigma_{i}$ is defined in (25), then there exists a diffeomorphsim $\left(z^{T}, \xi\right)^{T}=\phi(x, w)$ which transforms the extended system (18-20) into the proposed normal form (21-23).

Proof 1. Let $\Delta=\operatorname{span}\left\{\sigma_{1}, \ldots, \sigma_{n}\right\}$ be the distribution spanned by vector fields $\sigma_{i}$ for $1 \leq i \leq n$. As $\left[\sigma_{i}, \sigma_{j}\right]=0$ for $1 \leq i, j \leq n$, then thanks to Theorem 2.36 in Nijmeijer and Van der Schaft (1990), there exists a local diffeomorphism $\left(z^{T}, \xi\right)^{T}=\phi(x, w)$ such that

$$
\phi_{*}\left(\sigma_{i}\right)=\frac{\partial}{\partial z_{i}} \text { for } 1 \leq i \leq n .
$$

Now, let us consider the vector field $v=\phi_{*}^{-1}\left(\frac{\partial}{\partial \xi}\right)$. As $\left\{\sigma_{1}, \cdots, \sigma_{n}, \frac{\partial}{\partial w}\right\}$ forms a basis, then we can write

$$
v=r_{1}(x, w) \frac{\partial}{\partial w}+r_{2}(x, w) \sigma_{1}+\cdots+r_{n+1}(x, w) \sigma_{n}
$$

where $r_{1} \neq 0$. As $\left[\frac{\partial}{\partial z_{i}}, \frac{\partial}{\partial \xi}\right]=0$, then we have

$$
\left[\sigma_{i}, v\right]=\left[\phi_{*}^{-1}\left(\frac{\partial}{\partial z_{i}}\right), \phi_{*}^{-1}\left(\frac{\partial}{\partial \xi}\right)\right]=\phi_{*}^{-1}\left[\frac{\partial}{\partial z_{i}}, \frac{\partial}{\partial \xi}\right]=0
$$

In the following, we will show that $r_{1}(x, w)$ is only a function of the auxiliary variable $w$. Indeed, as $\left[\sigma_{i}, \sigma_{j}\right]=0$, a direct calculation gives:

$$
\left[\sigma_{i}, v\right]=\left(L_{\sigma_{i}} r_{1}\right) \frac{\partial}{\partial w}+r_{1}\left[\sigma_{i}, \frac{\partial}{\partial w}\right]+\sum_{j=1}^{n}\left(L_{\sigma_{i}} r_{j+1}\right) \sigma_{j}
$$


Moreover, by construction we have

$$
\sigma_{i}=\sum_{j=1}^{n} \mu_{i, j}(x, w) \frac{\partial}{\partial x_{i}}
$$

for $1 \leq i \leq n$, therefore we obtain $\left[\sigma_{i}, \frac{\partial}{\partial w}\right]=-\sum_{j=1}^{n} \frac{\partial \mu_{i, j}}{\partial w} \frac{\partial}{\partial x_{i}} \in \Delta$. Thus, it can be seen that $\left[\sigma_{i}, v\right]=0$ if and only if

$$
\left(L_{\sigma_{i}} r_{1}\right) \frac{\partial}{\partial w}=0
$$

and

$$
r_{1}\left[\sigma_{i}, \frac{\partial}{\partial w}\right]+\left(L_{\sigma_{i}} r_{2}\right) \sigma_{1}+\left(L_{\sigma_{i}} r_{3}\right) \sigma_{2}+\ldots+\left(L_{\sigma_{i}} r_{n+1}\right) \sigma_{n}=0
$$

Then one has $L_{\sigma_{i}} r_{1}=0$, which implies that $r_{1}(w)$ depend only on $w$.

Now, consider $\sigma_{n+1}=\frac{1}{r_{1}(w)} v=\frac{\partial}{\partial w}+\frac{r_{2}}{r_{1}} \sigma_{1}+\ldots+\frac{r_{n+1}}{r_{1}} \sigma_{n}$. This vector field fulfils $\left[\sigma_{i}, v\right]=r_{1}\left[\sigma_{i}, \sigma_{n+1}\right]=0$, and we have $d w\left(\sigma_{n+1}\right)=1$.

Note

$$
\sigma=\left[\sigma_{1}, \sigma_{2}, \cdots, \sigma_{n}, \sigma_{n+1}\right]
$$

and denote the set of the observability 1-forms of the extended system as:

$$
\theta_{e}=\left(d h, d L_{F} h, \cdots, d L_{F}^{n-1} h, d w\right)^{T}
$$

then one can calculate the following matrix:

$$
\Lambda=\theta_{e} \sigma=\left(\begin{array}{ccccccc}
0 & 0 & 0 & \ldots & 0 & l & * \\
0 & 0 & 0 & 0 & l \alpha_{n} & * & \vdots \\
0 & \vdots & 0 & \ddots & * & \vdots & * \\
\vdots & 0 & \frac{l \pi}{\alpha_{2} \alpha_{3}} & * & \vdots & * & * \\
0 & \frac{l \pi}{\alpha_{2}} & * & \ldots & * & * & * \\
l \pi & * & * & \ldots & * & * & * \\
0 & 0 & 0 & 0 & 0 & 0 & 1
\end{array}\right)
$$

It is clear that $\Lambda$ is invertible, thus one can define the following multi 1-forms:

$$
\omega=\left(\begin{array}{c}
\omega_{1} \\
\omega_{2} \\
\vdots \\
\omega_{n+1}
\end{array}\right)=\Lambda^{-1} \theta_{e}
$$

and one has $\omega_{i} \sigma_{j}=\delta_{i j}$ where $\delta_{i j}$ is the Kronecker's delta for $1 \leq i \leq n+1$ and $1 \leq j \leq n+1$.

Let $X$ and $Y$ be two vectors fields in $\left\{\sigma_{1}, \ldots, \sigma_{n}, \sigma_{n+1}\right\}$, then one has:

$$
d \omega_{i}(X, Y)=L_{Y} \omega_{i}(X)-L_{X} \omega_{i}(Y)-\omega_{i}[X, Y]
$$

Since $\omega_{i}(X)$ and $\omega_{i}(Y)$ are constant, one obtains:

$$
d \omega_{i}(X, Y)=-\omega_{i}[X, Y]
$$

Therefore, one has the equivalence between $[X, Y]=0$ and $d \omega_{i}=0$ for $1 \leq i \leq n+1$. Thus by Poincaré's lemma there exists $\phi=\left(\phi_{1}, \cdots, \phi_{n+1}\right)^{T}$ such that $\omega=d \phi:=\phi_{*}$.

Therefore, one can set:

$$
\omega\left(\sigma_{i}\right)=\phi_{*}\left(\sigma_{i}\right)=\frac{\partial}{\partial z_{i}}
$$

for $1 \leq i \leq n$ and $\phi_{*}\left(\sigma_{n+1}\right)=\frac{\partial}{\partial \xi}$.

Now, we analyze the influence of the diffeomorphism on the extended vector field $F$. For $1 \leq i \leq n-1$ one has:

$$
\begin{aligned}
\frac{\partial \phi_{*}(F)}{\partial z_{i}} & =\left[\frac{\partial}{\partial z_{i}}, \phi_{*}(F)\right]=\left[\phi_{*}\left(\sigma_{i}\right), \phi_{*}(F)\right] \\
& =\phi_{*}\left[\sigma_{i}, F\right]=\alpha_{i+1} \phi_{*} \sigma_{i+1} \\
& =\alpha_{i+1} \frac{\partial}{\partial z_{i+1}}
\end{aligned}
$$

So by integration one obtains $\dot{z}_{i}=\alpha_{i+1}(y) z_{i}+\beta_{i}(y)$ for $1 \leq i \leq$ $n-1$.

For the last two dynamics, we use the definition of $\omega$ given in (26), and after a straightforward calculation one obtains:

$$
\begin{aligned}
\omega_{n} & =d \phi_{n}=\frac{d h}{l(w)}+\rho(x) d w \\
\omega_{n+1} & =d \phi_{n+1}=d w
\end{aligned}
$$

where $\rho(x)$ is a function of $x$. The equation (28) implies that $\xi=\phi_{n+1}=w$.

Moreover, since $\omega_{n}$ is a closed 1-form, i.e. $d \omega_{n}=0$, so according to equation (27), $\rho(x)$ should be only a function of $y$ and $w$. Hence $z_{n}=\phi_{n}$ should be as well a function of the known variables $y$ and $w$. Therefore, one can denote a new output $\bar{y}$ in the following form:

$$
\phi_{n}(y, w)=z_{n}=C z
$$

and this implies that system (18-20) is transformed into (21-23).

Remark 1. - As $\omega=d \phi=\phi_{*}$, then the diffeomorphism can be obtained by integration: $z_{i}=\phi_{i}(x)=\int \omega_{i}$.

- If the vector field $\sigma_{n+1}$ is obtained by induction as $\sigma_{n+1}=$ $\left[\sigma_{n}, F\right]$, then $\frac{\partial \phi_{*}(F)}{\partial z_{n}}=\frac{\partial}{\partial \xi}$ and the normal form become $\dot{z}=$ $A(y) z+B(w)$, where the second term $B(w)$ will depend only on the auxiliary variable $w$.

- As in assumption of Lemma 3.1 in Boutat and Busawon (2011), it can be shown that $\left[\bar{\tau}_{i}, \bar{\tau}_{n}\right]-\varrho(y) \bar{\tau}_{i-1} \in$ $\operatorname{span}\left\{\bar{\tau}_{1}, \cdots, \bar{\tau}_{i-1}\right\}$ is the condition to ensure the existence of $\eta(y, w)$ and $l(w)$, where $\varrho(y)$ is only a function of $y$.

\section{Application to SEIR model}

\subsection{Calculation of $\tau$}

Considering the SEIR model described in (9-11), a simple calculation gives the associated observability 1-forms as follows:

$$
\begin{aligned}
& \theta_{1}=d x_{3} \\
& \theta_{2}=\varepsilon d x_{2}+\left(\gamma_{3}+2 \delta x_{3}\right) d x_{3} \\
& \theta_{3}=\varepsilon \beta x_{3} d x_{1}+\varepsilon\left(\gamma_{2}+\gamma_{3}+3 \delta x_{3}\right) d x_{2}+Q_{1} d x_{3}
\end{aligned}
$$


where $Q_{1}=\varepsilon \beta x_{1}+3 \varepsilon \delta x_{2}+\varepsilon q b+\gamma_{3}^{2}+6 \delta \gamma_{3} x_{3}+6 \delta^{2} x_{3}^{2}$.

Then the associated frame $\tau$ is given by:

$$
\begin{aligned}
& \tau_{1}=\frac{1}{\varepsilon \beta x_{3}} \frac{\partial}{\partial x_{1}} \\
& \tau_{2}=u \tau_{1}+\frac{1}{\varepsilon} \frac{\partial}{\partial x_{2}} \quad \text { where } u=-b+\gamma_{3}+\left(\delta+\gamma_{1}\right) x_{3}+\varepsilon \frac{x_{2}}{x_{3}} \\
& \tau_{3}=-\frac{p b}{\varepsilon} \frac{\partial}{\partial x_{1}}-\left(L_{f} u\right) \tau_{1}+u \tau_{2}+\frac{1}{\varepsilon}\left(\gamma_{2}+\delta x_{3}\right) \frac{\partial}{\partial x_{2}}+\frac{\partial}{\partial x_{3}}
\end{aligned}
$$

A straightforward calculation gives $\left[\tau_{1}, \tau_{2}\right]=\left[\tau_{1}, \tau_{3}\right]=0$ and

$$
\left[\tau_{2}, \tau_{3}\right]=Q_{2} \tau_{1}+\frac{1}{x_{3}} \tau_{2}
$$

where $Q_{2}=-\left(3 \delta+2 \gamma_{1}+\frac{2 \gamma_{2}-\gamma_{3}}{x_{3}}-3 \varepsilon \frac{x_{2}}{x_{3}^{2}}\right)$.

As $\left[\tau_{2}, \tau_{3}\right] \neq 0$, then the system (9-11) cannot be transformed into the observer form with output injection, but one can use them to construct a new frame $\bar{\tau}$.

\subsection{Calculation of $\bar{\tau}$}

To build $\bar{\tau}$, one needs to seek non vanishing functions $\alpha_{2}(y)$ and $\alpha_{3}(y)$ from $\tau$. Without loss of generality, one can always assume that $\alpha_{3}(y)=1$. The reason is that if $\alpha_{3}(y)$ is different to 1 in one normal form, one can always apply a diffeomorphism on the output $z_{3}=\int_{0}^{y} \frac{1}{\alpha_{3}(s)} d s$ which will make $\alpha_{3}(y)=1$ in the transformed normal form. Therefore, one needs only to determine $\alpha_{2}(y)$.

According to Zheng et al. (2007), one uses the following equation:

$$
\left[\tau_{2}, \tau_{3}\right]=\lambda(y) \tau_{2} \quad \bmod \tau_{1}
$$

with $\lambda(y)=\frac{d \alpha_{2}(y)}{d y} \frac{1}{\alpha_{2}(y)}$ to determine $\alpha_{2}(y)$. Then one has:

$$
\frac{d \alpha_{2}(y)}{d y} \frac{1}{\alpha_{2}(y)}=\frac{1}{x_{3}}=\frac{1}{y}
$$

which yields $\alpha_{2}(y)=y=x_{3}$.

Consequently, one has $\alpha_{2}=x_{3}, \alpha_{3}=1$ and $\pi=\alpha_{2} \alpha_{3}=x_{3}$. Then from (6) one obtains:

$$
\begin{aligned}
\bar{\tau}_{1} & =x_{3} \tau_{1}=\frac{1}{\varepsilon \beta} \frac{\partial}{\partial x_{1}} \\
\bar{\tau}_{2} & =\frac{1}{x_{3}}\left[\bar{\tau}_{1}, f\right]=\frac{1}{\varepsilon \beta x_{3}}\left(-b+\gamma_{1} x_{3}\right) \frac{\partial}{\partial x_{1}}+\frac{1}{\varepsilon} \frac{\partial}{\partial x_{2}} \\
\bar{\tau}_{3} & =\left[\bar{\tau}_{2}, f\right]=\left(-p b \beta+\frac{\left(-b+\gamma_{1} x_{3}\right)^{2}}{x_{3}}-b \varepsilon \frac{x_{2}}{x_{3}^{2}}\right) \bar{\tau}_{1} \\
& +\frac{1}{\varepsilon}\left(\gamma_{2}-b+\left(\delta+\gamma_{1}\right) x_{3}\right) \frac{\partial}{\partial x_{2}}+\frac{\partial}{\partial x_{3}}
\end{aligned}
$$

A straightforward calculation gives $\left[\bar{\tau}_{1}, \bar{\tau}_{2}\right]=\left[\bar{\tau}_{1}, \bar{\tau}_{3}\right]=0$ and $\left[\bar{\tau}_{2}, \bar{\tau}_{3}\right]=-2 \frac{b}{x_{3}^{2}} \bar{\tau}_{1}$. One can see again that the commutativity condition for the new frame $\bar{\tau}$ is not satisfied, and according to the third point of Remark 1 the functions $\eta(y, w)$ and $l(w)$ exist.

\subsection{Calculation of $\sigma$}

In this step, we will seek an auxiliary dynamics $\dot{w}=\eta(y, w)$ and a non zero function $l(w)$ which fulfill the condition of Theorem 1 . For this, set $\sigma_{1}=l(w) \bar{\tau}_{1}=\frac{l}{\varepsilon \beta} \frac{\partial}{\partial x_{1}}$, then one has:

$$
\begin{aligned}
\sigma_{2}= & \frac{1}{x_{3}}\left[\sigma_{1}, F\right]=\frac{1}{x_{3}}\left(l H-\eta l^{\prime}\right) \sigma_{1}+\frac{l}{\varepsilon} \frac{\partial}{\partial x_{2}} \\
\sigma_{3}= & {\left[\sigma_{2}, F\right]=\left(-L_{F}\left(\frac{1}{x_{3}}\left(l H-\eta l^{\prime}\right)\right)-\beta p b\right) \sigma_{1}+\left(l H-\eta l^{\prime}\right) \sigma_{2} } \\
& +\left(\frac{l}{\varepsilon}\left(\gamma_{2}+\delta x_{3}\right)-\frac{l^{\prime}}{\varepsilon} \eta\right) \frac{\partial}{\partial x_{2}}+l \frac{\partial}{\partial x_{3}}
\end{aligned}
$$

where $H=\left(-b+\gamma_{1} x_{3}\right)$. Finally, one gets:

$$
\left[\sigma_{2}, \sigma_{3}\right]=\left(\frac{l}{\varepsilon} L_{\frac{\partial}{\partial x_{2}}}\left(-L_{F}\left(\frac{1}{x_{3}}\left(l H-\eta l^{\prime}\right)\right)-l L_{\frac{\partial}{\partial x_{3}}}\left(\frac{1}{x_{3}}\left(l H-\eta l^{\prime}\right)\right)\right) \sigma_{1}\right.
$$

Therefore, $\left[\sigma_{2}, \sigma_{3}\right]=0$ implies that:

$$
\frac{l}{\varepsilon} L_{\frac{\partial}{\partial x_{2}}}\left(-L_{F}\left(\frac{1}{x_{3}}\left(l H-\eta l^{\prime}\right)\right)-l L_{\frac{\partial}{\partial x_{3}}}\left(\frac{1}{x_{3}}\left(l H-\eta l^{\prime}\right)\right)=0\right.
$$

which is equivalent to:

$$
-l b+l^{\prime}\left(-\eta+x_{3} \eta_{y}^{\prime}\right)=0
$$

where $\eta_{y}^{\prime}=\frac{\partial \eta}{\partial y}$ and $l^{\prime}=\frac{d l}{d w}$.

As $l$ is only a function of $w$ then $-\eta+x_{3} \eta_{y}^{\prime}$ is only a function of $w$. Consequently, the function $\eta(w, y)$ has the following form: $\eta=\kappa_{1}(w) y+\kappa_{2}(w)$. Then, according to (29) one has:

$$
l b+l^{\prime} \kappa_{2}(w)=0
$$

which implies $l(w)=e^{-\int_{0}^{w} \frac{b}{k_{2}(s)} d s}$. To simplify the calculations, we take $\eta=-\kappa(w) H=-\kappa(w)\left(\gamma_{1} x_{3}-b\right)$, i.e. $\kappa_{1}(w)=-\kappa(w) \gamma_{1}$ and $\kappa_{2}(w)=\kappa(w) b$.

Then, the corresponding frame $\sigma$ is as follows:

$$
\begin{aligned}
\sigma_{1} & =\frac{l}{\varepsilon \beta} \frac{\partial}{\partial x_{1}}, \quad \sigma_{2}=\frac{l}{\varepsilon} \frac{\partial}{\partial x_{2}} \\
\sigma_{3} & =-\frac{p b}{\varepsilon} l \frac{\partial}{\partial x_{1}}+\frac{\gamma_{2}+b+\left(\delta-\gamma_{1}\right) x_{3}}{\varepsilon} l \frac{\partial}{\partial x_{2}}+l \frac{\partial}{\partial x_{3}}
\end{aligned}
$$

One can check that $\left[\sigma_{1}, \sigma_{2}\right]=\left[\sigma_{1}, \sigma_{3}\right]=\left[\sigma_{2}, \sigma_{3}\right]=0$. To complete the dimension of the frame, one should find $\sigma_{4}$ which commutes with $\sigma_{i}$ for $1 \leq i \leq 3$. For this, one can choose:

$\sigma_{4}=\frac{l^{\prime} x_{1}}{l} \frac{\partial}{\partial x_{1}}+\frac{l^{\prime}}{l}\left(x_{2}+\frac{\left(\delta-\gamma_{1}\right)}{2 \varepsilon} x_{3}^{2}\right) \frac{\partial}{\partial x_{2}}+\frac{l^{\prime} x_{3}}{l} \frac{\partial}{\partial x_{3}}+\frac{\partial}{\partial w}$

which makes $\left[\sigma_{4}, \sigma_{i}\right]=0$ for $1 \leq i \leq 3$.

\subsection{Determination of diffeomorphism}

After determining the auxiliary dynamics, one can calculate the observability 1 -forms $\theta_{e}=\left[d h, d L_{F} h, d L_{F}^{2}, d w\right]^{T}$, then one obtains:

$$
\Lambda=\theta_{e} \sigma=\left(\begin{array}{cccc}
0 & 0 & l & \frac{l^{\prime}}{l} x_{3} \\
0 & l & \Lambda_{23} & \Lambda_{24} \\
x_{3} l & l\left(\gamma_{2}+\gamma_{3}+3 \delta x_{3}\right) & \Lambda_{33} & \Lambda_{34} \\
0 & 0 & 0 & 1
\end{array}\right)
$$


where:

$$
\begin{aligned}
& \Lambda_{23}=\left(\gamma_{2}+b+\left(\delta-\gamma_{1}\right) x_{3}\right) l+\left(\gamma_{3}+2 \delta x_{3}\right) l \\
& \Lambda_{24}=\frac{l^{\prime}}{2 l}\left(2 \varepsilon x_{2}+2 \gamma_{3} x_{3}+\left(5 \delta-\gamma_{1}\right) x_{3}^{2}\right) \\
& \Lambda_{33}=\left(\gamma_{2}+\gamma_{3}+3 \delta x_{3}\right)\left(\gamma_{2}+b+\left(\delta-\gamma_{1}\right) x_{3}\right) l-p b \varepsilon \beta x_{3} l+Q_{1} l \\
& \Lambda_{34}=\frac{l^{\prime}}{l}\left(x_{2}+\frac{\left(\delta-\gamma_{1}\right)}{2 \varepsilon} x_{3}^{2}\right) \varepsilon\left(\gamma_{2}+\gamma_{3}+3 \delta x_{3}\right)+\varepsilon \beta x_{3} \frac{l^{\prime} x_{1}}{l}+\frac{l^{\prime} x_{3}}{l} Q_{1}
\end{aligned}
$$

Thus one obtains $\omega=\Lambda^{-1} \theta_{e}=d z$ which yields the diffeomorphism given in (16) allowing to transform the system (9-10-1113) into the form (17), where:

$$
\begin{aligned}
B_{1}(y, w)= & b \beta\left(\frac{1}{l}\left(p\left(b+\gamma_{3}\right)-q \varepsilon\right) y+\frac{p}{l}\left(\delta-\gamma_{1}\right) y^{2}\right)+\frac{b \beta \varepsilon}{l} \\
B_{2}(y, w)= & -\beta b p y+\frac{1}{l}\left(\delta^{2}+\frac{1}{2} \gamma_{1}^{2}-\frac{3}{2} \delta \gamma_{1}\right) y^{3}- \\
& \frac{1}{l}\left(\delta\left(\gamma_{2}+\gamma_{3}+\frac{3}{2} b\right)-2 \gamma_{1} \gamma_{3}-\frac{3}{2} b \gamma_{1}\right) y^{2}- \\
& \frac{1}{l}\left(b\left(\gamma_{2}+\gamma_{3}+b-q \varepsilon\right)+\gamma_{2} \gamma_{3}\right) y \\
B_{3}(y, w)= & \frac{1}{l}\left(3 b+\gamma_{2}+\gamma_{3}\right) y+\frac{3}{2 l}\left(\delta-\frac{5}{3} \gamma_{1}\right) y^{2}
\end{aligned}
$$

\subsection{Simulation results}

For the simulation, we chose $\kappa(w)=\frac{\sin ^{2}(a w)}{(a w)^{2}}$ where $\left.a \in\right] 0,1[$, and the same parameters of the SEIR model as those in Li and Muldowney (1996) are used, i.e. $N=141, b=0.221176 / N$, $\delta=0.002, p=0.8, q=0.95, \beta=0.05, \varepsilon=0.05, r=0.003$. The initial conditions are $S(0)=140, E(0)=0.01, I(0)=0.02$ and $N(0)=141$. The simulation results are presented in Fig. $1-4$.

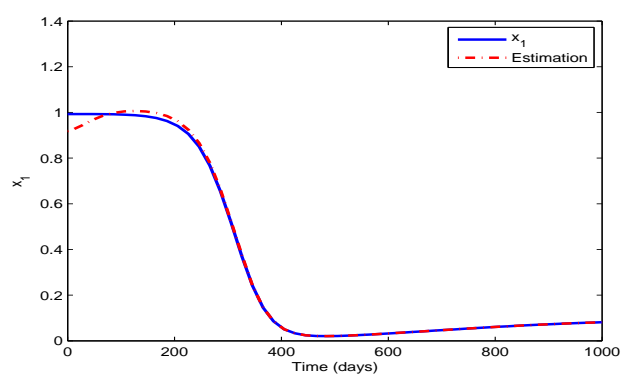

Figure 1: Estimation of $x_{1}$

\section{Conclusion}

This paper introduced a new extended output depending normal form, which mixes both the extended normal form and the output depending normal form. This new normal form enables to design a simple high gain observer. Sufficient condition was given in order to guarantee the existence of a diffeomorphism which can be used to transform the extended dynamical systems into the proposed normal form. Finally, the proposed result was applied to the SEIR model to estimate the evolution of different populations during a contagious disease.

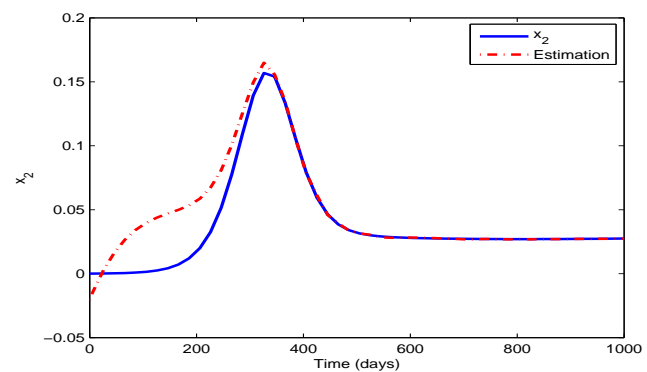

Figure 2: Estimation of $x_{2}$

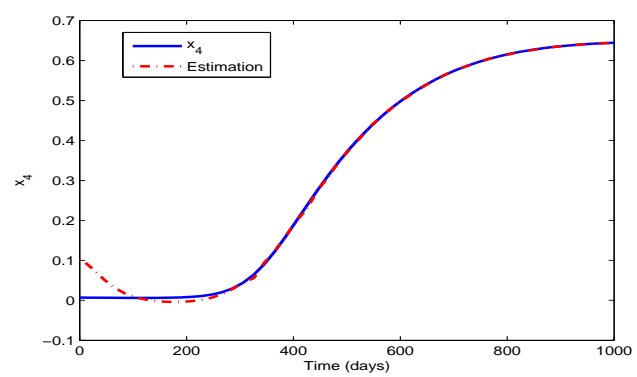

Figure 3: Estimation of $x_{4}$

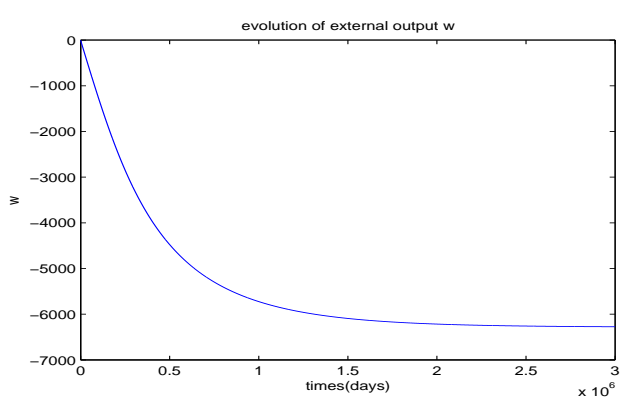

Figure 4: Boundedness of auxiliary dynamics $(w)$

Acknowledgements: The author thanks the referees for their careful reading and useful comments that allowed a substantial improvement of this paper.

Back, J., Yu, K. T., Seo, J. H., 2006. Dynamic observer error linearization. Automatica, 42 (12), 2195-2200.

Bestle, D., Zeitz, M., 1983. Canonical form observer design for nonlinear time varying systems. Internatio $38,419-431$.

Boutat, D., 2007. Geometrical conditions for observer error linearization via $\int 0,1, \ldots,(n-2)$. In: 7 th IFAC Symposium on Nonlinear Control Systems Nolcos'07,

Boutat, D., Benali, A., Hammouri, H., Busawon, K., 2009. New algorithm for observer error linearization with a diffeomorphism on the outputs. Automatica 45 (10), 2187-2193.

Boutat, D., Busawon, K., 2011. On the transformation of nonlinear dynamical systems into the extended nonlinear observable canonical form. International Journal of Control, 84 (1), 94-106.

Busawon, K., Farza, M., Hammouri, H., 1998. Asimple observer for a class of nonlinear systems. Applied Mathematics Letters 11 (3), 27-31.

Guay, M., oct 2002. Observer linearization by output-dependent time-scale transformations. IEEE Transactions on Automatic Control, 47 (10), 1730 -1735 . 
Hou, M., Pugh, A., 1999. Observer with linear error dynamics for nonlinear multi-output systems. Systems \& Control Letters, 37, 1-9.

Iggidr, A., 2006. Controllability, observability and stability of mathematical models. Mathematical Models. In Encyclopedia of Life Support Systems (EOLSS). Developed under the auspices of the UNESCO, Eolss Publishers, Oxford, UK.

Jouan, P., 2003. Immersion of nonlinear systems into linear systems modulo output injection. SIAM Journal on Control and Optimisation, 41(6), 17561778.

Keller, H., 1987. Nonlinear observer design by transformation into a generalized observer canonical form. International Journal of Control, 46(6), 19151930.

Krener, A., Isidori, A., 1983. Linearization by output injection and nonlinear observers. Systems \& Control Letters, 3 (1), 47-52.

Krener, A., Respondek, W., 1985. Nonlinear observers with linearizable error dynamics. SIAM Journal on Control and Optimization, 23 (2), 197-216.

Li, M. Y., Muldowney, J. S., 1996. A geometric approach to global-stability problems. SIAM Journal on Mathematical Analysis, 27, 1070-1083.

Lopez, M., Plestan, F., Glumineau, A., 1999. Linearization by completely generalized input ouput injection. Kybernetika, 35, 793-802.

Lynch, A., Bortoff, S., jun 2001. Nonlinear observers with approximately linear error dynamics: the multivariable case. IEEE Transactions on Automatic Control, 46 (6), 927-932.

Marino, R., Tomei, P., 1996. Nonlinear control design: geometric, adaptive and robust.

Nijmeijer, H., Van der Schaft, A., 1990. Nonlinear dynamical control systems. Springer-Verlag.

Noh, D., Jo, N., Seo, J., 2004. Nonlinear observer design by dynamic observer error linearization. IEEE Transactions on Automatic Control, 49 (10), 17461753.

Phelps, A., 1991. On constructing nonlinear observers. SIAM Journal on Control and Optimmisation, 29, 516-534.

Respondek, W., Pogromsky, A., Nijmeijer, H., 2004. Time scaling for observer design with linearizable error dynamics. Automatica, 40 (2), 277-285.

Rudolph, J., Zeitz, M., 1994. A block triangular nonlinear observer normal form. Systems \& Control Letters, 23 (1), 1-8.

Wang, Y., Lynch, A., 2009. Observer design using a generalized time-scaled block triangular observer form. Systems \& Control Letters, 58, 346-352.

Wang, Y., Lynch, A., 2010. Multiple time scalings of a multi-output observer form. IEEE Transactions on Automatic Control, 55, 966-971.

Xia, X., Gao, W., 1989. Nonlinear observer with linearizable error dynamics. SIAM Journalon Control and Optimization, 27, 199-216.

Yang, J., Back, J., Seo, J., Shim, H., 2010. Reduced-order dynamic observer error linearization. In: Nonlinear Control Systems,. pp. 915-920.

Yang, J., Back, J., Seo, J. H., 2011. A complete solution to a simple case of dynamic observer error linearization: New approach to observer error linearization. IEICE transactions on fundamentals of electronics, communications and computer sciences, 94 (1), 424-429.

Zheng, G., Boutat, D., Barbot, J., 2007. A single output dependent observability normal form. SIAM Journal on Control and Optimization, 46 (6), 22422255. 